\title{
Periodistas: El acceso al mercado laboral
}

\author{
Caro González, Francisco Javier \\ Jiménez Marín, Gloria \\ Universidad de Sevilla
}

\begin{abstract}
Resumen
El presente estudio analiza la situación profesional de los Licenciados en Periodismo en España. Para ello esta investigación sigue la lógica del proceso de acceso al mercado laboral, es decir, se estudia, en primer lugar, la oferta de egresados y las demandas del sector; a continuación, se analiza la forma en la que un titulado en la Licenciatura de Periodismo accede al mercado laboral y el tiempo que tarda en hacerlo; y por último, se examinan los medios de comunicación en los que encuentran trabajos estos egresados y las condiciones de estos puestos: formación requerida, tipología de contrato y sueldo.
\end{abstract}

\begin{abstract}
The current study analyzes the professional situation of the Graduated in Journalism in Spain. For it, this investigation follows the logic of the access process the professional market, that is to say, it studies, in the first place, the supply of Graduated and the demands of the sector; next, it analyzes the form in which a titleholder in the Degree of Journalism accedes to the professioinal market and the time that takes in doing it; and finally, the mass media in which they find a jobs these Graduated persons and the conditions of these positions are examined: required formation, tipology of contract, and wage.
\end{abstract}

Palabras clave: Demanda laboral, Licenciatura en Periodismo, Mercado de trabajo, Oferta laboral, Periodistas.

Key words: Degree in Journalism, Journalists, Labor demand, Labor supply, Market of work.

\section{LA FORMACIÓN UNIVERSITARIA}

a formación universitaria es insuficiente. En general, los profesionales del mundo del periodismo consideran que la formación recibida en las facultades y centros universitarios es inadecuada y no se ajusta a las necesidades reales que exige la profesión. En este sentido, la opinión de Díaz Nosty (1998: 60) es tajante, ya que afirma que los planteamientos de las facultades de comunicación están "desfasados y escasamente dinámicos", puesto que las necesidades del mercado avanzan mucho más rápidamente que los planes de estudios.

Asimismo, muchas son las opiniones acerca de la idoneidad de formar a futuros periodistas, es decir, muchas son las voces que a día de hoy aún se cuestionan la necesidad de formación universitaria en Periodismo, lo que desprestigia a la profesión. 
Respecto al primer punto, hay que reconocer que realmente existe un desfase entre el mundo empresarial y el universitario; por su parte, también se da una necesidad de establecer algún tipo de mecanismo que ayude a una unión Universidad-Empresa que suponga una base para la formación real e idónea para los futuros periodistas. Una muestra de estas carencias formativas en esta área es el incremento de Másters y Cursos de Experto para complementar la formación recibida durante los años de carrera.

Respecto al segundo de los puntos comentados, la captación, utilización, manejo, transformación, difusión y control de la información exigen una enorme responsabilidad en todo el proceso de la profesión periodística, y es lo suficientemente complejo como para exigir una formación superior de calidad que no se limite a los antiguos cursos de formación profesional y que, por su parte, no permita el intrusismo profesional.

Las Asociaciones de Prensa llevan muchos años intentando sacar adelante un estatuto de la profesión que, hoy por hoy no existe. Tan sólo la prensa periódica no diaria dispone de un convenio marco estatal que, en ningún caso, puede compararse con un estatuto o bases de actuación para la prensa en general. En los demás sectores (prensa diaria, agencias informativas, radiodifusión y televisión) no existe. Sólo en prensa diaria se extendió el convenio de empresa del El Norte de Castilla a convenio estatal como primer paso para negociar un acuerdo marco, pero, aún así, no existe un estatuto serio y con rigor a nivel nacional.

En el año 1999 se celebró en Sevilla el Primer Congreso de Periodistas de Andalucía en el que se pusieron de manifiesto las lagunas e insuficiencias quee los profesionales del sector tenían (y tienen) y se proponía la creación de un Colegio de Periodistas que protegiera los intereses de la profesión. Asimismo, otras de estas propuestas pretendían fortalecer la cualificación de los profesionales de los medios de comunicación y la dignificación de los centros de formación universitarios. En este sentido, también se discutió acerca de la posibilidad de "creación de instituciones de cultura democrática que ordenen el panorama de un hipersector que ya tiene un carácter nuclear, en las sociedades más avanzadas" (Díaz-Nosty, 1999: 40).

La convención de Bolonia, firmada por la mayoría de los Estado de la Unión Europea y terceros países, y la futura convergencia hacia el EEES (Espacio Europeo de Educación Superior), plantean una orientación de la formación mucho más profesional para el estudiante universitario, lo que supone una gran oportunidad para aproximar la actual enseñanza universitaria a la realidad empresarial con la que se topan los egresados de esta titulación. Se trata, por tanto, de formar a profesionales autónomos con capacidad de aprender y de adaptarse a los cambios. En este trabajo, a partir de diversos estudios que analizan las características laborales del sector, se realizan algunas reflexiones acerca de las características de la oferta de empleo a los periodistas. 


\section{REALIDAD LABORAL VS. CONTENIDOS FORMATIVOS: CONCILIA- CIÓN Y DISCORDIA}

\subsection{La percepción de los estudiantes de Periodismo.}

La primera cuestión que hay que analizar es la percepción que se tiene de los egresados de la Licenciatura en Periodismo. El Libro Blanco de los Estudios de Comunicación (ANECA, 2005) analiza diversos estudios realizados en los que se muestran opiniones acerca de la Licenciatura en Periodismo; entre ellos, cabe destacar los datos extraídos de esta titulación en la Facultad de Santiago de Compostela, en el que se evidencia que la estimación de la carrera de Periodismo por parte de los Licenciados en el período 1995-2002 es la siguiente:

- El $40 \%$ opina que es buena, frente a un $6 \%$ que la califica como mala.

- Casi un 50\% sitúa la formación con una calificación de regular

- Un 2\% la sitúa en los extremos: muy mala o muy buena.

Por su parte, en la Universidad Autónoma de Barcelona (UAB), el nivel de satisfacción con la licenciatura es menos favorable:

- El 64,7\% opina que está "ni mucho ni poco" satisfecho con los estudios recibidos.

- El 30,5\% se decanta por un "satisfactorio".

- En los extremos se encuentran un $3 \%$ poco satisfecho y un $2 \%$ muy satisfecho.

Con respecto a los aspectos mejor valorados por los licenciados, en la Facultad de Santiago de Compostela destaca que más de un $56 \%$ del alumnado opina que son las prácticas, cuestión que, como se analiza más adelante, es una de las carencias más importantes de los egresados desde el punto de vista de los empleadores. Este porcentaje es seguido muy lejos por las materias específicas $(8,5 \%)$ o el pensamiento crítico $(6,2 \%)$. En el otro extremo, el $31,5 \%$ es el profesorado. Si bien es posible que el efecto horn haya hecho su aparición en las valoraciones, y los profesores muy malos hayan impedido una valoración objetiva; este dato debe ser un elemento de reflexión acerca del papel del profesorado. El segundo aspecto peor valorado es la teoría $(24,6 \%)$. Estos datos puede verse en la tabla 1. 
Tabla 1:

VALORACIONES DE LOS EGRESADOS DE LA TITULACIÓN DE PERIODISMO EN LA USC

\begin{tabular}{|l|c|c|}
\hline & Lo mejor valorado & Lo peor valorado \\
\hline Prácticas en empresas & $56 \%$ & \\
\hline Materias específicas & $8,5 \%$ & \\
\hline Pensamiento crítico & $6,2 \%$ & \\
\hline Profesorado & & $31,5 \%$ \\
\hline Teoría & & $24,6 \%$ \\
\hline
\end{tabular}

Elaboración propia. Fuente: ANECA, 2005.

\subsection{Adecuación de la actual formación en Periodismo a las nece- sidades reales del mundo laboral}

Existe un desajuste entre la actual formación universitaria que reciben los alumnos y el mundo profesional que se encuentran éstos cuando egresan de la Licenciatura. Las universidades buscan formar personas con conocimientos profesionales que contribuyan al crecimiento de la sociedad, forman a personas con espíritu crítico; por su parte, el mundo empresarial exige máquinas intelectuales engrasadas que incrementen la productividad con escasa inversión.

La Fundación Conocimiento y Desarrollo (CYD) (2005) realizó un estudio entre 404 empresas para indagar en qué medida los titulados universitarios cuentan con aptitudes que solicitan las empresas a los egresados de la titulación en Periodismo. De este modo, se analizan las principales carencias que estas entidades encuentran en el alumnado que se introduce en el mundo profesional: la formación práctica y las habilidades directivas conforman estas insuficiencias. También tienen un peso importante las carencias en habilidades de comunicación e idiomas.

Otro estudio realizado entre profesionales de la información por la Faculta de CC. de la Información de Santiago de Compostela (2004) estudia la valoración subjetiva que los licenciados hacían sobre las actitudes y habilidades más valoradas por sus superiores. El orden de priorización de las exigencias puede verse en la tabla 2: 
Tabla 2:

ACTITUDES Y HABILIDADES MÁS VALORADAS POR SUS SUPERIORES SEGÚN LOS LICENCIADOS EN PERIODISMO

\begin{tabular}{|c|l|c|}
\hline 1. & Rapidez en la redacción de informaciones & 7,64 \\
\hline 2. & Buena redacción de las informaciones & 7,54 \\
\hline 3. & Dominio de nuevas tecnologías & 7,53 \\
\hline 4. & Circunscripción a los espacios y tiempos asignados & 7,43 \\
\hline 5. & Identificación con la línea editorial de la empresa & 7,25 \\
\hline 6. & Capacidad para proponer nuevos temas & 7,18 \\
\hline 7. & Proximidad a los poderes (político, económico, etc.) & 7,03 \\
\hline 8. & Aceptación sin discusión de las órdenes & 7,02 \\
\hline 9. & Publicación sólo de informaciones suficientemente contrastadas & 6,69 \\
\hline 10. & Investigación de asuntos de actualidad & 6,41 \\
\hline 11. & Fomento de debates sobre temas de actualidad & 6,07 \\
\hline 12. & Respeto al código deontológico de la profesión & 6,04 \\
\hline 13. & Proximidad a las asociaciones ciudadanas & 5,86 \\
\hline 14. & Proposición de nuevos enfoques a temas asignados & 5,58 \\
\hline
\end{tabular}

Fuente: Facultad de Ciencias de la Comunicación de Santiago de Compostela, 2004.

Las actitudes y habilidades mejor valoradas que perciben los directores y jefes de sección acerca de los periodistas son las relativas al trabajo operativo del mismo, es decir, las destinadas al incremento de la eficacia en las rutinas de producción informativa. Otro dato que llama poderosamente la atención es que por encima de 7, se encuentra la afirmación "Aceptación sin discusión de las órdenes", que da idea de un estilo de dirección predominante, autoritario y con escasa capacidad de orientación al cambio y a la innovación (el aspecto menos valorado es la "Proposición de nuevos enfoques a temas asignados").

Del mismo modo, llama la atención la posición número 12 acerca del "respeto al código deontológico de la profesión", cuando supuestamente éste es uno de los pilares sobre los que se constituye la labor del periodista, y una de las tareas fundamentales del proceso formativo. En este sentido en el puesto número 5 se ubica "la identificación con la línea editorial", muy por encima de la deontología profesional, cuestión que subraya el conflicto que existe en las empresas periodísticas entre el enfoque ideológico y el enfoque mercantilista del empresario y las consideraciones éticas propias de la profesión. 


\section{LA INSERCIÓN LABORAL}

Es importante analizar cómo ha ido evolucionando el número de Licenciados en España durante los últimos años. En el gráfico 1 se puede observar cómo esta cifra se ha mantenido estable durante los últimos años produciéndose un pico el curso 98-99. Éste se debe al cambio de planes de estudios, donde la mayoría de los centros pasaron de planes de estudios de 4 a 5 años.

\section{Gráfico 1:}

\section{EVOLUCIÓN DEL NÚMERO DE LICENCIADOS EN CC. DE LA INFORMACIÓN EN ESPAÑA}

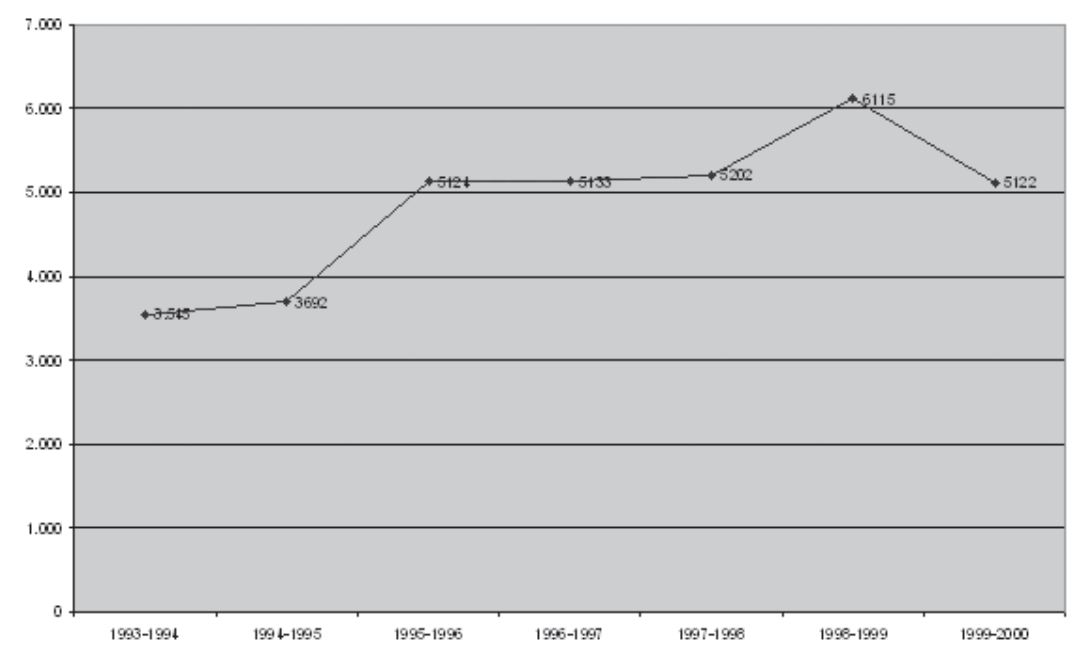

Fuente: ANECA (2005)

De este modo, en España, un total de 26.706 alumnos egresaron durante este período de tiempo compitiendo por alcanzar alguno de los puestos fijos que existen en las empresas de comunicación en la fecha del estudio. Atendiendo al perfil del egresado en el año 2000 puede concluirse que, en el $65 \%$ de los casos, se trata de una mujer residente en Madrid, comunidad que concentra más del $55 \%$ de los licenciados durante el mismo año. En la comunidad autónoma en la que tienen más peso las mujeres es en las Islas Baleares, donde representan más del $80 \%$, frente a otras comunidades como Andalucía, en el que apenas se llegan al $60 \%$.

Pero el número de estudiantes de Periodismo ha sufrido un estancamiento, que puede deberse a que esté dejando de ser una titulación de "moda" y los estudiantes estén tomando conciencia de la realidad profesional a la que se enfrentan en el mercado laboral y, por ello, se decanten por otras opciones formativas como los estudios en Comunicación Audiovisual o Publicidad y RR.PP. que van ganando terreno al Periodismo. 
Analizando los datos del mercado laboral se puede comprobar a qué contexto profesional se enfrentan los egresados en la actualidad. Y es que, según un estudio de la empresa de consultoría de recursos humanos Círculo de Progreso, con datos de 2001, la carrera de Periodismo es de las que tiene un mayor potencial de inserción laboral. Este indicador se establece relacionando el porcentaje de ofertas de trabajo dirigido a titulados sin experiencia en relación con el total de puestos de trabajo ofrecidos. Según este informe, el $41,42 \%$ de las ofertas que reciben los periodistas no requieren experiencia. Esto facilita el acceso a la profesión pero también da lugar a una situación laboral más precaria que en otras profesiones, ya que las condiciones de acceso suelen ser contratos temporales, de prácticas o becarios que prolongan sus duración durante varios años, lo que, a su vez, conlleva a una caída de estímulos por parte del trabajador.

En relación a las prácticas, los estudiantes de periodismo realizan sus primeras incursiones en el mundo laboral como becarios en las empresas de comunicación. La diferencia con otras titulaciones es que, una vez terminada la carrera, el becario sigue realizando prácticas durante varios años. La carrera lógica constatada pasa por constituirse como freelances cuando ha transcurrido un determinado período de tiempo desempeñando la misma labor.

Durante las vacaciones estivales es usual cubrir los puestos de los redactores con estudiantes en prácticas que suplen por completo las tareas de los redactores profesionales. De esta manera es práctica habitual la realización de contratos temporales que encubren un trabajo a tiempo completo o la contratación de Licenciados para categorías muy inferiores a las que le corresponden a la titulación, como es el caso de contratos con la categoría de Auxiliares Administrativos para Licenciados en Periodismo.

La encuesta CHEERS (García-Montalvo, 2002: 3) indica que a los cuatro años después de terminar la carrera, la tasa de actividad de los licenciados en Periodismo era del $90,9 \%$, con una tasa de paro del 7,8\%. El 1,3\% restante representa a los que continuaron estudiando y a los que se retiraron del mercado laboral. Pese a lo que podría parecer de positivo, este dato sitúa a los periodistas en un nivel intermedio de entre 33 titulaciones españolas analizadas, ocupando el puesto 15, muy por debajo de las tasas de actividad de los Ingenieros Industriales, Arquitectos o Ingenieros Agrónomos que cuenta con un 99'3, 99 y 98\%, respectivamente y por tanto, unas tasas de paro nulas. Pero también quedan alejados de los graduados en Magisterio que cuentan con un $71,7 \%$ de tasa de actividad y un $16,8 \%$ de paro; o de los Licenciados en Geografía e Historia, que tienen un $77,7 \%$ de actividad y un $24,3 \%$ de paro; o los Ldos. en Biología que tienen un $80,7 \%$ de egresados activos y un $25,2 \%$ de parados. Bel (2004: 306$)$ es más pesimista y califica en el desempleo de los Licenciados en Periodismo como "angustioso y realmente alarmante en este sector".

En definitiva, los titulados en Periodismo no tienen dificultades en acceder al mercado laboral, ya que el potencial de inserción laboral es muy elevado y apenas se exige experiencia para trabajar siendo, a su vez, el tiempo medio de acceso a 
un puesto de trabajo inferior a los cuatro meses. Sin embargo, esta facilidad para encontrar trabajo no garantiza la permanencia en él ni es indicativa de la calidad del mismo, sino que existen indicios de la precariedad laboral del sector que, frecuentemente, contrata a Licenciados con una categoría profesional muy inferior a la que les pertenece.

\section{EL MERCADO DE TRABAJO DE LOS PERIODISTAS}

Un Licenciado en Periodismo tiene muchas posibilidades de encontrar ya que la versatilidad de la formación y las exigencias de la Sociedad de la Información favorecen que el abanico de posibilidades laborales de estos titulados sea muy amplio. De hecho, los titulados no desembarcan sólo en medios de comunicación aunque siguen siendo éstas las que acogen al mayor número de Licenciados.

En función del tipo de empresa se pueden distinguir varios tipos de empresa (García y Zofio: 2003):

- Prensa escrita

- Radio y televisión

- Cine y vídeo

- Publicidad.

En el Gráfico 2 puede observarse la evolución del número de trabajadores de las distintas empresas de comunicación:

\section{Gráfico 2: EVOLUCIÓN DEL NÚMERO DE ASALARIADOS}

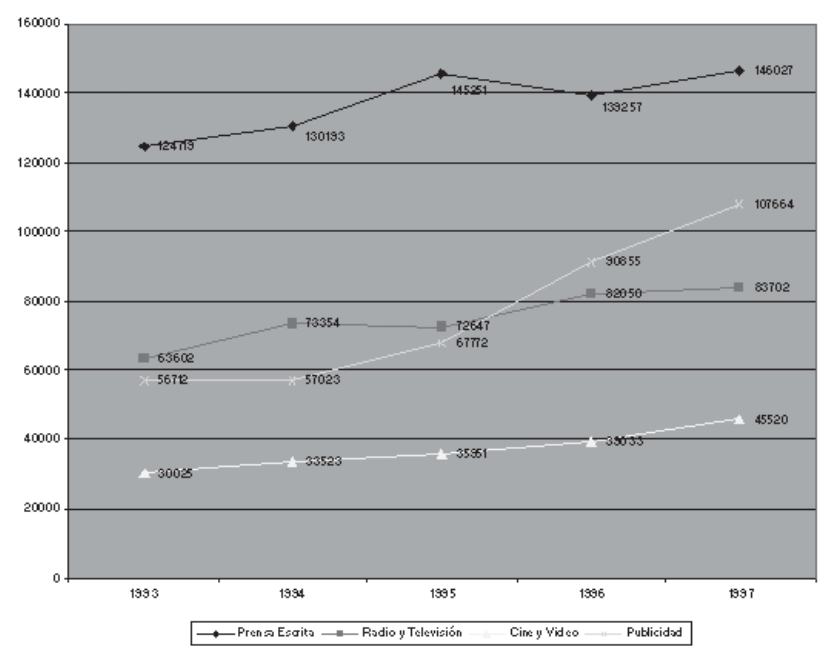

Elaboración propia. Fuente de datos: GARCíA y ZOFíO (2003). 
Como puede observarse, la actividad empresarial que acapara más empleados es la prensa escrita. Sin embargo, la actividad que ha experimentado el mayor crecimiento es la de publicidad con un 90,2 \% en los cinco años considerados. La prensa escrita supone más del $38 \%$ de los empleos de los periodistas, y la publicidad, el $28 \%$. Es importante resaltar que, en muchas universidades no se imparte la formación específica de Publicidad y RR.PP., lo que hace que muchos periodistas ejerzan en este sector. Posiblemente estos datos se hayan visto modificados con la potenciación del sector audiovisual, y la televisión y el cine y el video hayan ganado cierto terreno en la capacidad de emplear a los egresados.

El estudio de la Facultad de Comunicación de Santiago de Compostela sobre las salidas profesionales de la Licenciatura en Periodismo (1995-2002) expone algunos datos interesantes aunque no son extrapolables directamente al resto de España. En él se hace alusión a que tres cuartas partes de los Licenciados durante esos ocho cursos están trabajando como periodistas; por su parte, uno de cada diez, trabaja en otros ámbitos y el 8'4 está en paro. Este último dato es un poco más alto que el de la encuesta CHEERS, datos que, en principio, pueden deberse a la realidad socio-laboral-comunicativa de Galicia. En comparación, el estudio de Santiago de Compostela contempla una media de desempleo de los Licenciados durante los ocho cursos analizados del 8,4\% mientras que, en el caso de la encuesta CHEERS, a los cuatro años de finalizada la carrera, la tasa de paro es del $7,8 \%$. Este desempleo, a su vez, afecta más a la población masculina, con un $10,9 \%$, que a la femenina que posee un paro del $7,5 \%$.

Respecto al autoempleo, se constata que tiene poco peso en la profesión periodística. En Galicia, el porcentaje de autoempleados no alcanza el 3\%, mientras que en el resto de España, según la encuesta CHEERS, el porcentaje sigue siendo muy bajo, de un $6 \%$, en comparación con otras profesiones, como es el caso de los Arquitectos, que en el $72,6 \%$ de los casos son trabajadores por cuenta propia. Este dato permite establecer dos hipótesis plausibles: por un lado, los Licenciados en Periodismo carecen de espíritu emprendedor que puede ser debida a una escasa formación empresarial; por otro, la estructura de las empresas de comunicación impide la incorporación al sector de nuevas empresas si no es formando parte de los grandes grupos existentes.

Frente al nuevo Espacio Europeo de Educación Superior, constituye un reto interesante el hecho de fomentar la iniciativa emprendedora de los egresados para que sean ellos mismos los que dinamicen el sector con una oferta atractiva. Esto cada día es más posible teniendo en cuenta las posibilidades que brindan las TIC, y la importancia que están adquiriendo los gestores de información, personas capaces de desenvolverse en Internet y seleccionar y ordenar las fuentes informativas. 


\section{SUELDO}

En las III Jornadas de Empleo de la Universidad Autónoma de Madrid (UAM), los responsables de la sección de economía de medios de comunicación destacaron las principales características del empleo en el ámbito de los medios de comunicación en materia salarial:

- Una elevada estratificación salarial

- Una remuneración muy desequilibrada.

Sin embargo, atendiendo a la encuesta CHEERS, se observa cómo los periodistas se encuentran en una posición intermedia en relación a otros Licenciados. Los periodistas tienen unos ingresos anuales medios de 14.780 • frente al sueldo medio de los Ingenieros de Caminos que cobran 29.660 • o el de los arquitectos, con 26.190. Por su parte, están mejor pagados que los Licenciados en Biología, que cobran una media de 11.900 anuales, o los Licenciados en Pedagogía, con 12.430 al año. Asimismo, el sueldo medio de los Licenciados en Ciencias Sociales es de $16.000 \cdot$, lo que implica que los Licenciados en Periodismo reciben un salario por debajo de la media de su grupo.

El estudio de Santiago de Compostela, además, presenta unos resultados desalentadores:

- El $37 \%$ de los licenciados cobran entre 600 y 900 al mes (entre 8400 y 12600 - considerando 14 meses).

- El $27 \%$ entre 900 y $1200 \cdot(12600-16800)$.

- El 20\% menos de 600 - y sólo el $16 \%$ más de 1200 .

Por lo tanto, en base a estos datos se extrae que más de la mitad de Licenciados en Periodismo tiene una nómina mensual inferior a los 900 euros, nómina fácilmente superable por otras profesiones en las que no se exija una base de Licenciatura.

Del mismo modo, en función de los soportes, los periodistas que trabajan en radio son los peor pagados, ya que, por término medio no llegan a un salario de 600 euros netos al mes, mientras que los que ejercen en gabinetes de comunicación o en televisión se acercan a los 800 y 900 euros, respectivamente. Sin embargo, el Libro Blanco de los Estudios de Comunicación añade un dato ligeramente positivo acerca de la percepción que tienen los periodistas de su salario: Ofrece datos aportados por el Colegio de Periodistas de Cataluña sobre una encuesta realizada a sus colegiados en 2004 del que se extrae que el $47,5 \%$ se considera mal remunerado, frente a un $52,5 \%$ que opina que no. La AGP (2000) abogaba por establecer en los convenios de medios de comunicación un salario base mínimo para los periodistas equivalente a 2,5 veces el salario mínimo interprofesional, salario que, en la muchos de los casos no se alcanza ya que se realizan contratos por debajo de la categoría profesional que al periodista en cuestión le corresponde. 


\section{TIPO DE CONTRATO}

Según los resultados de la encuesta CHEERS (García-Motalvo, 2002: 4), cuatro años después de haberse graduado, el 51,9\% de los licenciados en Periodismo tienen contrato indefinido. Las profesiones con más contratos indefinidos son los arquitectos (84\%) y los que menos, los médicos (22,1\%). En Cataluña, el 67,5\% de los periodistas encuestados tiene contrato indefinido, y ésta es una tendencia creciente. En el otro lado de la balanza se observa que los contratos temporales han decrecido más de un $13 \%$ (porcentaje acumulado), en los últimos doce años. La encuesta realizada en Galicia entre los Licenciados en Periodismo de los años 1995-2001 es muy desalentadora puesto que indica que tan sólo el 31,4\% de los Licenciados tiene trabajo fijo. La situación que más se da es la de contratos temporales en un $43,6 \%$ de los casos. Por su parte, un $7,1 \%$ de los Licenciados trabajan sin contrato.

En relación a actividades profesionales, es la prensa la que ofrece mayor estabilidad con un $68 \%$ de contratos fijos. En el lado opuesto, está la televisión, donde el porcentaje de contratos fijos no llega al $12 \%$. Esto se debe a la propia naturaleza del sector que trabaja por programas $y / 0$ temporadas y sobre todo realizan contratos por obra y artísticos.

Bel $(2004,306)$ y la AGP $(2000)$ opinan que el sector está sumido en precariedad laboral "por la elevada tasa de paro, la falta de regulación y las trampas legales existentes, que permiten a las empresas contar con una legión de periodistas dispuestos a firmar contratos temporales en condiciones leoninas".

\section{CONCLUSIONES}

De todo lo anteriormente expuesto se extrae que los recién Licenciados en Periodismo se encuentran con un entorno profesional nada complaciente, al que resulta bastante fácil acceder, y cuyas condiciones laborales son bastante precarias. Los sueldos, en general, son bajos, y la inestabilidad es bastante elevada.

La mayoría de las empresas del sector pueden ser calificadas de pequeñas empresas, rozando la microempresa (menos de 10 trabajadores) y esto dificulta alcanzar unas buenas condiciones laborales ya que es difícil especializarse, las promociones en las empresas no son posibles por su estructura reducida, etc.

Por su parte, los empresarios regulan la profesión periodística sin que los empleados puedan ejercer presión. Existe una unión muy escasa en este colectivo y salvo algunos colegios de periodistas más activos y algunas asociaciones de la prensa reivindicativas, prácticamente no existe ningún elemento de poder que se contraponga al de la patronal. Es necesario que exista una mayor unión de los periodistas que los colegios profesionales y las asociaciones de la prensa jueguen un papel activo para garantizar los derechos de estos profesionales vitales para informar, formar y entretener al público objetivo, responsable, en último caso, de la compra o consumo de esos productos informativos. 


\section{BIBLIOGRAFÍA:}

AGP (2000): "Radiografía de la profesión periodística". En Opinar. http//www.opinar.net $(05 / 04 / 00)$

ANECA (2005): Libro Blanco. "Títulos de Grado en Ciencias de la Comunicación". www.aneca.com

BANCAJA (2002): "La situación laboral de los graduados españoles". En Capital Humano, n. 21, julio.

BEL MALLÉN, J.I. (2004): "La formación, un desafío permanente para la información". Libro Blanco de la Prensa Diaria, 2004. AEDE: Madrid.

CINCO DÍAS (2003): "Un tercio de la oferta de empleo reclama recién licenciados", en Cinco Días (versión digital), 28 febrero 2003, Madrid.

COELLO I BRUFAU, Joaquím (2006): "Los peligros de Bolonia: El Espacio Europeo de Educación Superior". En Nuevo Trabajo, domingo 16 de abril de 2006.

CPC (2004). Llibre blanc de la professió periodística a Catalunya. CPC.

DíAZ NOSTY, B. (1999): La Comunicación en Andalucía 1999/ Situación y tendencias.

GARCÍA GRACIA, M. I., ZOFÍO PRIETO, J. L. (2003): La dimensión sectorial de la Industria de la Cultura y el Ocio en España (1993-1997). Iberautor. Madrid.

JIMÉNEZ AGUILERA, J.; MONTERO GRANADOS, R.; SÁNCHEZ CAMPILLO, J. (2003): Educación Superior y Empleo: la Situación de los Jóvenes Titulados en Europa. La Encuesta CHEERS. Universidad de Granada

MURCIANO, Marcial (2005). "La enseñanza del periodismo, nuevos desafío internos y externos". En Cuadernos de Periodistas, Diciembre de 2005.

PÉREZ DE PABLOS, S. (2005): “¿Qué opinan las empresas de los recién licenciados?”. En El País, jueves 24 de febrero de 2005.

PLAZA, T. y TÉBAR, N. (2002): "Licenciados de Ciencias de la Comunicación en el año 2000". Estudio encargado por el Decanato de la Facultad de Ciencias de la Comunicación de la Universidad Autónoma de Barcelona. Bellaterra (Barcelona).

SOMBRÍA PÉREZ, A. (2003): Seguimiento de los alumnos titulados en la Universidad SEK - Segovia. Universidad SEK. Segovia.

SORIANO, J. (Dir.) (2004): Llibre blanc de la professió periodística a Catalunya: informe de la recerca cualitativa (borrador). Collegi de Periodistes de Catalunya y Universitat Autònoma de Barcelona. Barcelona.

TÚÑEZ, M., PÉREZ, M. GÓMEZ, R. (2004): Da Facultade aou Mercado Laboral: estudo e análise das saídas profesionais da licenciatura de Xornalismo (1995-2002). Facultade de Ciencias da Comunicación, Universidade de Santiago de Compostela, Santiago de Compostela. 